\title{
THE PARALLEL DIFFUSION COEFFICIENT AND ADIABATIC FOCUSING OF COSMIC-RAY PARTICLES
}

\author{
YURI E. LITVINENKO \\ Department of Mathematics, University of Waikato, P.B. 3105, Hamilton, New Zealand \\ Received 2011 August 15; accepted 2011 September 30; published 2011 December 29
}

\begin{abstract}
In this paper, the problem of focused particle transport is revisited. A description in terms of a system of stochastic differential equations, completely equivalent to the Fokker-Planck equation, is suggested. The coefficient for spatial diffusion parallel to the mean magnetic field is calculated. The case of isotropic pitch angle scattering and weak focusing is analyzed in detail. The disagreement between a recent analysis by Shalchi and other treatments of the same problem is discussed.
\end{abstract}

Key words: cosmic rays - diffusion - magnetic fields

\section{INTRODUCTION}

The transport of energetic particles in turbulent cosmic magnetic fields is typically studied using the Fokker-Planck equation (see Schlickeiser 2011 for a recent systematic derivation). When the particle pitch angle distribution is close to the isotropic distribution, the Fokker-Planck equation can be integrated with respect to pitch angle to yield a simpler diffusion equation for the particle density (e.g., Hasselmann \& Wibberenz 1970). Investigations of cosmic-ray transport in interplanetary space, for instance, typically employ the Parker transport equation (e.g., Parker 1965; Gleeson \& Axford 1967; Jokipii \& Parker 1970). Description in terms of diffusion is valid as long as the scale of density variation is larger than the scattering mean free path.

Magnetic fields are often nonuniform in space plasmas. The conservation of the second adiabatic invariant dictates that particle pitch angles tend to focus toward the magnetic field lines. Unless the mean free path is negligibly small compared with the magnetic field focusing length, adiabatic focusing significantly modifies particle transport along the mean magnetic field (Roelof 1969; Earl 1976; Kunstmann 1979). Equations of focused particle transport have been used repeatedly to interpret cosmic-ray particle data (e.g., Bieber et al. 1986, 2002; Dröge et al. 2006; Le Roux et al. 2007; Sáiz et al. 2008; Le Roux \& Webb 2009). Adiabatic focusing also leads to an additional convective term in momentum space (Schlickeiser \& Shalchi 2008; Litvinenko \& Schlickeiser 2011), which can describe a potentially important mechanism of focused particle acceleration (Schlickeiser 2009; Schlickeiser et al. 2011).

The value of the coefficient $\kappa_{\|}$for spatial diffusion parallel to the mean magnetic field is particularly useful in quantitative description of the transport of energetic charged particles. When focusing can be ignored, an analytical formula for $\kappa_{\|}$in terms of the Fokker-Planck coefficient $D_{\mu \mu}$ for pitch angle scattering is well known (Jokipii 1966; Hasselmann \& Wibberenz 1968, 1970; Kulsrud \& Pearce 1969; Earl 1974).

The parallel diffusion coefficient taking into account adiabatic focusing has been evaluated by several independent methods: the parallel streaming inferred from an exact steady solution to the Fokker-Planck equation (Earl 1981; Beeck \& Wibberenz 1986), the Born approximation (Bieber \& Burger 1990), and the adjoint Green's function technique (Kóta 2000). There appears to be no disagreement among these methods within their common range of applicability.
Surprisingly, the results of a recent application of the Kubo (1957) formalism to the problem of focused transport (Shalchi 2011) disagree with the previous treatments of the problem.

Determining the correct dependence of the parallel diffusion coefficient on the focusing length is of obvious interest, and so the purpose of this paper is to reexamine the diffusive limit of focused particle transport. In addition to comparison of the previous results in a simple case of isotropic pitch angle scattering and weak focusing, a new independent method for deriving $\kappa_{\|}$is presented. The new description is formulated in terms of a system of stochastic differential equations equivalent to the Fokker-Planck equation.

\section{PREVIOUS RESULTS FOR THE PARALLEL DIFFUSION COEFFICIENT}

The Fokker-Planck equation for particle transport including effects of pitch angle scattering and adiabatic focusing is given by

$$
\frac{\partial f_{0}}{\partial t}+\mu v \frac{\partial f_{0}}{\partial z}+\frac{v}{2 L}\left(1-\mu^{2}\right) \frac{\partial f_{0}}{\partial \mu}=\frac{\partial}{\partial \mu}\left(D_{\mu \mu} \frac{\partial f_{0}}{\partial \mu}\right)
$$

(e.g., Roelof 1969; Earl 1981). Here, $f_{0}$ is the distribution function of energetic particles (gyrotropic phase-space density per unit length of magnetic line), $t$ is the time, $\mu$ is the particle pitch angle cosine, $v$ is the particle speed, $z$ is the distance along the mean magnetic field $B, L=-B /(\partial B / \partial z)$ is the focusing length of the magnetic field, and $D_{\mu \mu}$ is the Fokker-Planck coefficient for pitch angle scattering.

As a concrete illustration, consider the Fokker-Planck equation in the case of isotropic pitch angle scattering and a constant focusing length:

$$
D_{\mu \mu}=D_{0}\left(1-\mu^{2}\right),
$$

where $D_{0}=$ const and $L=$ const.

The parallel diffusion coefficient is conventionally written as

$$
\kappa_{\|}=\frac{1}{3} v \lambda,
$$

where $\lambda$ is the scattering mean free path. In the absence of focusing $(L=\infty)$, the standard expression for the mean free path $\lambda_{0}$ in terms of $D_{\mu \mu}$ is

$$
\lambda_{0}=\frac{3 v}{8} \int_{-1}^{1} \frac{\left(1-\mu^{2}\right)^{2}}{D_{\mu \mu}} d \mu=\frac{v}{2 D_{0}}
$$


(e.g., Equation (29) in Earl 1974 or Equation (3) in Beeck \& Wibberenz 1986), and so $\kappa_{\|}=v^{2} /\left(6 D_{0}\right)$ if $L=\infty$.

Turning now to focused transport $(L<\infty)$, consider for simplicity the weak-focusing limit, $\lambda_{0} / L \ll 1$, so that only the leading-order term in a small parameter $\lambda_{0} / L$ needs to be calculated. Now Equation (26) in Earl (1981) in the isotropic scattering case $q=1$, Equation (14) in Beeck \& Wibberenz (1986), Equation (54) in Bieber \& Burger (1990), or Equation (52) in Kóta (2000) all lead to the same expression for $\lambda=\lambda(L)$ :

$$
\frac{\lambda}{\lambda_{0}}=\left(1-\frac{1}{15} \frac{\lambda_{0}^{2}}{L^{2}}+\cdots\right) .
$$

In addition to deriving a general expression for $\lambda$, Beeck $\&$ Wibberenz (1986) explicitly analyzed the case of isotropic scattering; the equation above is their Equation (16b). Equation (35) in Shalchi (2011), however, gives a different expression:

$$
\frac{\lambda}{\lambda_{0}}=\left(1-\frac{2}{5} \frac{\lambda_{0}^{2}}{L^{2}}+\cdots\right) .
$$

Shalchi (2011) does not discuss the earlier calculations by Earl (1981), Beeck \& Wibberenz (1986), and Kóta (2000). Shalchi (2011), however, seriously misrepresents the work of Bieber \& Burger (1990) by claiming that Equation (40) in Bieber $\&$ Burger (1990) leads to the dependence $\lambda / \lambda_{0} \approx 1-\lambda_{0} / L$ when the magnetic helicity $\sigma=0$ (see discussion after Equation (43) in Shalchi 2011). In fact, setting $\sigma=0$ in Equation (40) of Bieber \& Burger (1990) gives $\kappa_{\|}=v \lambda_{0} / 3$. This means simply that $\left(\lambda / \lambda_{0}-1\right)$ is of order $\lambda_{0}^{2} / L^{2}$ in the approximation in which their Equation (40) was derived. Bieber \& Burger (1990) were primarily interested in the effect of a nonzero helicity $\sigma$ on $\kappa_{\|}$in the context of solar modulation, and so they kept only first-order terms in their approximation (see discussion before Equation (24) in Bieber \& Burger 1990). Hence Equation (40) in Bieber \& Burger (1990) is correct to a first order in the small parameter $\lambda_{0} / L$, and the assertion by Shalchi (2011) is unfounded. Bieber \& Burger (1990) clearly recognized that second-order terms must be taken into account if $\sigma=0$. As noted above, Equation (54), derived by Bieber \& Burger (1990) in the context of solar particle transport, leads to Equation (5), in perfect agreement with the calculations by Earl (1981), Beeck \& Wibberenz (1986), and Kóta (2000). Notably, Kóta (2000) pointed out that the expressions for $\kappa_{\|}$derived by Beeck \& Wibberenz (1986) and Bieber \& Burger (1990) are equivalent, as long as $D_{\mu \mu}$ is an even function of $\mu$, which implies $\sigma=0$.

Forman (1977) was the first to apply the Kubo (1957) formalism to the problem of particle transport in a uniform mean magnetic field. Both Kóta (2000) and Shalchi (2011) applied this formalism to the case of focused transport (compare Equation (41) in Kóta 2000 with Equations (11) and (12) in Shalchi 2011). Kóta (2000) obtained an eigenfunction expansion of the space-integrated adjoint Green's function and showed that the lowest-mode eigenvalue is $v_{0}=0$ (Equation (47) in Kóta 2000). Hence, each term in the expansion, except the $k=0$ term, decays exponentially in time. Physically, this means that the memory of initial conditions is not lost if the initial angular distribution happens to be the lowest-mode eigenfunction of a scattering operator.

Therefore, the complete loss of memory of the initial angular distribution, postulated in Equation (13) of Shalchi (2011), appears to be a dubious assumption (cf. Equation (48) in Kóta 2000).

\section{DESCRIPTION IN TERMS OF STOCHASTIC DIFFERENTIAL EQUATIONS}

To clarify the arguments leading to the standard expression for the parallel diffusion coefficient, it may be useful to derive a formula for $\kappa_{\|}$with a new independent method. The idea is to avoid solving the Fokker-Planck equation explicitly for the distribution function. Instead, particle transport can be described in terms of the pitch angle distribution moments, determined from a system of stochastic differential equations (Conway et al. 1998; Conway 2000; Litvinenko 2009). While simulations using stochastic differential equations have been used repeatedly to obtain solutions to the Fokker-Planck equation (e.g., Jokipii \& Owens 1975; Fichtner et al. 1996; Zhang 1999; Pei et al. 2010; Strauss et al. 2011), the analytical description brings out an important relationship between the diffusive and streaming aspects of an evolving particle distribution and emphasizes the key assumption of rapid relaxation of the angular distribution to a nonisotropic equilibrium.

The method is based on the complete equivalence of the Fokker-Planck equation to a system of stochastic differential equations (e.g., Gardiner 2009, chap. 4). Equation (1) can be rewritten as

$$
\begin{aligned}
\frac{\partial f}{\partial t}= & -\frac{\partial}{\partial z}(\mu v f)-\frac{\partial}{\partial \mu}\left[\frac{v}{2 L}\left(1-\mu^{2}\right) f+\frac{\partial D_{\mu \mu}}{\partial \mu} f\right] \\
& +\frac{\partial^{2}}{\partial \mu^{2}}\left(D_{\mu \mu} f\right),
\end{aligned}
$$

where $f=\exp (z / L) f_{0}$. As stated previously, $L=$ const is assumed for simplicity. Application of the Ito stochastic calculus leads to the following system, which retains the full information about the evolution of the particle distribution function:

$$
\begin{gathered}
d z=\mu v d t, \\
d \mu=\left[\frac{v}{2 L}\left(1-\mu^{2}\right)+\frac{\partial D_{\mu \mu}}{\partial \mu}\right] d t+\sqrt{2 D_{\mu \mu}} d W(t),
\end{gathered}
$$

where $d W$ represents a Wiener process with the zero mean and the variance equal to $t$. In the case of isotropic pitch angle scattering, $D_{\mu \mu}=D_{0}\left(1-\mu^{2}\right)$, the previous equation becomes

$$
d \mu=\left[\frac{v}{2 L}\left(1-\mu^{2}\right)-2 D_{0} \mu\right] d t+\sqrt{2 D_{0}\left(1-\mu^{2}\right)} d W .
$$

The diffusion limit applies if the particle distribution is slowly varying, so that a characteristic evolution timescale is much greater than the pitch angle scattering time. Hence the local angular distribution can be assumed to relax quickly to a steady near-isotropic state, and so $d \mu \approx 0$. Then Equation (10) can be rewritten approximately as

$$
\mu d t=\frac{v}{4 D_{0} L}\left(1-\mu^{2}\right) d t+\sqrt{\frac{1-\mu^{2}}{2 D_{0}}} d W
$$

Substituting this into Equation (8) yields

$$
d z=\frac{v^{2}}{4 D_{0} L}\left(1-\mu^{2}\right) d t+v \sqrt{\frac{1-\mu^{2}}{2 D_{0}}} d W .
$$

In contrast to Equation (8), Equation (12) makes it clear that particle transport can be approximately represented as a 
combination of coherent streaming and diffusion. The first term on the right-hand side of Equation (12) describes streaming, whereas the second term describes a diffusion process with variance $v^{2}\left(1-\mu^{2}\right) /\left(2 D_{0}\right)$. Therefore the spatial diffusion coefficient, obtained by averaging over $\mu$, is given by

$$
\kappa_{\|}=\frac{v^{2}}{4 D_{0}}\left\langle 1-\mu^{2}\right\rangle,
$$

where the angle brackets denote an average over the particle distribution.

Equations for the distribution moments $\left\langle\mu^{k}\right\rangle$ of any order are obtained using Ito's formula (e.g., Gardiner 2009, chap. 4). The first few moment equations are as follows:

$$
\begin{gathered}
\frac{d\langle\mu\rangle}{d t}=\frac{v}{2 L}-2 D_{0}\langle\mu\rangle-\frac{v}{2 L}\left\langle\mu^{2}\right\rangle, \\
\frac{d\left\langle\mu^{2}\right\rangle}{d t}=2 D_{0}+\frac{v}{L}\langle\mu\rangle-6 D_{0}\left\langle\mu^{2}\right\rangle-\frac{v}{L}\left\langle\mu^{3}\right\rangle, \\
\frac{d\left\langle\mu^{3}\right\rangle}{d t}=6 D_{0}\langle\mu\rangle+\frac{3 v}{2 L}\left\langle\mu^{2}\right\rangle-12 D_{0}\left\langle\mu^{3}\right\rangle-\frac{3 v}{2 L}\left\langle\mu^{4}\right\rangle .
\end{gathered}
$$

Consistent with the approximation $d \mu \approx 0$ in Equation (10), all derivatives $d\left\langle\mu^{k}\right\rangle / d t$ can be set to zero. It is clear from the moment equations that the distribution anisotropy is indeed weak if the focusing is weak, for instance $\langle\mu\rangle \approx v /\left(6 D_{0} L\right) \ll 1$ if $v /\left(D_{0} L\right) \ll 1$. Hence, the system can be solved either by iterations or simply by using $\left\langle\mu^{4}\right\rangle \approx \frac{3}{5}\left\langle\mu^{2}\right\rangle$. The resulting expression for $\left\langle\mu^{2}\right\rangle$ in the weak-focusing limit is given by

$$
\left\langle\mu^{2}\right\rangle=\frac{1}{3}\left(1+\frac{1}{30} \frac{v^{2}}{D_{0}^{2} L^{2}}+\cdots\right) .
$$

Note for clarity that the assumption of rapid relaxation of the distribution to a near-isotropic state is not valid in the strong focusing limit $\lambda_{0} / L \gg 1$.

Finally, combining Equations (13) and (17) yields

$$
\kappa_{\|}=\frac{v^{2}}{6 D_{0}}\left(1-\frac{1}{60} \frac{v^{2}}{D_{0}^{2} L^{2}}+\cdots\right),
$$

which is identical to Equation (5) since $\kappa_{\|}=v \lambda / 3$ and $\lambda_{0}=v /\left(2 D_{0}\right)$. Therefore, the description in terms of stochastic differential equations agrees with the calculations of $\kappa_{\|}$by Earl (1981), Beeck \& Wibberenz (1986), Bieber \& Burger (1990), and Kóta (2000), but disagrees with an expression for $\kappa_{\|}$obtained by Shalchi (2011).

The applicability of the present method is not limited to the case of isotropic pitch angle scattering. As a simple illustration, consider the Fokker-Planck equation with an arbitrary pitch angle scattering coefficient $D_{\mu \mu}$ in a uniform mean magnetic field $(L=\infty)$. The key approximation $d \mu \approx 0$ in Equation (9) leads to an expression for $\mu d t$ that can be substituted into Equation (8) to yield

$$
d z=\frac{v\left(1-\mu^{2}\right)^{2}}{2 D_{\mu \mu}} \frac{\partial}{\partial \mu}\left[\frac{D_{\mu \mu}}{\left(1-\mu^{2}\right)}\right] d t+\frac{v\left(1-\mu^{2}\right)}{\sqrt{2 D_{\mu \mu}}} d W,
$$

and so

$$
\kappa_{\|}=\frac{v^{2}}{4}\left\langle\frac{\left(1-\mu^{2}\right)^{2}}{D_{\mu \mu}}\right\rangle \text {. }
$$

In the absence of focusing, the averaging corresponds to integration with respect to $\mu$. Therefore,

$$
\kappa_{\|}=\frac{v^{2}}{8} \int_{-1}^{1} \frac{\left(1-\mu^{2}\right)^{2}}{D_{\mu \mu}} d \mu
$$

which is the standard result (e.g., Beeck \& Wibberenz 1986 and references therein). Comparison with the earlier derivations (e.g., Jokipii 1966; Hasselmann \& Wibberenz 1970; Beeck \& Wibberenz 1986) suggests that the new method can be quite powerful and elegant in a more general problem of particle transport. For instance, the dependence of particle transport coefficients on both adiabatic focusing and magnetic helicity can be determined (Y. E. Litvinenko 2011, in preparation).

\section{DISCUSSION}

The independent calculations of the parallel diffusion coefficient $\kappa_{\|}$by Earl (1981), Beeck \& Wibberenz (1986), Bieber \& Burger (1990), and Kóta (2000), as well as the analysis of this paper, agree with one another within their common range of applicability, specifically in the case of isotropic pitch angle scattering and weak focusing. By contrast, a recent calculation by Shalchi (2011) leads to an expression for $\kappa_{\|}$that differs from all the others in the first nonvanishing order in the small parameter $\lambda_{0} / L$.

It seems inevitable that at least one of the methods is erroneous, and it would be useful to understand the reasons for the discrepancy. As argued above, postulating the loss of memory of the initial angular distribution (Equation (13) of Shalchi 2011) is a questionable assumption that appears to contradict an eigenfunction expansion of the space-integrated adjoint Green's function containing the lowest-mode eigenvalue $v_{0}=0$ (Equation (47) in Kóta 2000). Shalchi (2011) claims that he "presented a reliable tool for computing diffusion coefficients along the mean magnetic field" whereas "the previous results... allow only a very crude estimation of the parallel diffusion coefficient." The validity of these claims, however, seems extremely doubtful.

The present description of diffusive transport in the weak focusing limit in terms of stochastic differential equations emphasizes the key requirement of strong pitch angle scattering that causes the angular distribution function to relax rapidly to a near-isotropic state. Another advantage is that the description makes it easier to understand some results of the method of adjoint Green's functions.

For example, Kóta (2000) showed that each term in the eigenfunction expansion except the $k=0$ term decays exponentially in time. The corresponding result in the present approach is obtained directly from the stochastic differential Equation (10) since it represents a partial differential equation with an exact steady solution describing an anisotropic particle distribution $f(\mu) \sim \exp \left(\lambda_{0} \mu / L\right)$. An initial distribution of this form would remain unaltered at any time $t>0$.

Kóta (2000) also showed that any initial distribution of a constant spatial gradient evolves after a few scattering times to a distribution moving with a constant speed $\kappa_{\|} / L$ (Equation (54) in Kóta 2000; see also Earl 1981). In the present description, the same connection between diffusion and streaming follows immediately from comparison of the two terms on the right-hand side of Equation (12).

This work was supported in part by NASA grant NNX08AG44G. I am grateful to Professor R. Schlickeiser 
(Ruhr-Universität Bochum, Germany) for drawing my attention to the problem of focused particle transport.

\section{REFERENCES}

Beeck, J., \& Wibberenz, G. 1986, ApJ, 311, 437

Bieber, J. W., \& Burger, R. A. 1990, ApJ, 348, 597

Bieber, J. W., Dröge, W., Evenson, P. A., et al. 2002, ApJ, 567, 622

Bieber, J. W., Evenson, P. A., \& Pomerantz, M. A. 1986, J. Geophys. Res., 91, 8713

Conway, A. J. 2000, A\&A, 362, 383

Conway, A. J., MacKinnon, A. L., Brown, J. C., \& McArthur, G. 1998, A\&A, 331, 1103

Dröge, W., Kartavykh, Y. Y., Klecker, B., \& Mason, G. M. 2006, ApJ, 645, 1516

Earl, J. A. 1974, ApJ, 193, 231

Earl, J. A. 1976, ApJ, 205, 900

Earl, J. A. 1981, ApJ, 251, 739

Fichtner, H., Le Roux, J. A., Mall, U., \& Rucinski, D. 1996, A\&A, 314, 650

Forman, M. A. 1977, Ap\&SS, 49, 83

Gardiner, C. W. 2009, Stochastic Methods: A Handbook for the Natural and Social Sciences (Berlin: Springer)

Gleeson, L. J., \& Axford, W. I. 1967, ApJ, 149, L115

Hasselmann, K., \& Wibberenz, G. 1968, Z. Geophys., 34, 353

Hasselmann, K., \& Wibberenz, G. 1970, ApJ, 162, 1049
Jokipii, J. R. 1966, ApJ, 146, 480

Jokipii, J. R., \& Owens, A. J. 1975, J. Geophys. Res., 80, 1209

Jokipii, J. R., \& Parker, E. N. 1970, ApJ, 160, 735

Kóta, J. 2000, J. Geophys. Res., 105, 2403

Kubo, R. 1957, J. Phys. Soc. Japan, 12, 570

Kulsrud, R., \& Pearce, W. P. 1969, ApJ, 156, 445

Kunstmann, J. 1979, ApJ, 229, 812

Le Roux, J. A., \& Webb, G. M. 2009, ApJ, 693, 534

Le Roux, J. A., Webb, G. M., Florinski, V., \& Zank, G. P. 2007, ApJ, 662, 350

Litvinenko, Y. E. 2009, A\&A, 496, 129

Litvinenko, Y. E., \& Schlickeiser, R. A. 2011, ApJ, 732, L31

Parker, E. N. 1965, Planet. Space Sci., 13, 9

Pei, C., Bieber, J. W., Burger, R. A., \& Clem, J. 2010, J. Geophys. Res., 115, A12107

Roelof, E. C. 1969, in Lectures in High Energy Astrophysics, ed. H. Ögelman \& J. R. Wayland (NASA SP-199; Washington, DC: NASA), 111

Sáiz, A., Ruffolo, D., Bieber, J. W., Evenson, P., \& Pyle, R. 2008, ApJ, 672, 650

Schlickeiser, R. 2009, Mod. Phys. Lett. A, 24, 1461

Schlickeiser, R. 2011, ApJ, 732, 96

Schlickeiser, R., Artmann, S., \& Zöller, C. 2011, Nucl. Phys. B: Proc. Suppl., 212,181

Schlickeiser, R., \& Shalchi, A. 2008, ApJ, 686, 292

Shalchi, A. 2011, ApJ, 728, 113

Strauss, R. D., Potgieter, M. S., Büsching, I., \& Kopp, A. 2011, ApJ, 735, 83

Zhang, M. 1999, ApJ, 512, 409 